\title{
Introduction to Session 4: New Biofuels and Biomass Chemicals
}

\author{
Hans Blaschek • Akwasi Boateng
}

Published online: 25 March 2009

(C) Humana Press 2009

This session will specialize in discussions of new fuels and chemicals emerging in the research community. Approaches include development of bio-gasoline, mixed alcohols, merging of thermochemical processes with biological conversion, as well as new targets for biomass-derived chemicals. While ethanol and biodiesel receive the most attention in the media, researchers continue to make progress in developing new fuels and chemicals from renewable sources and via some very interesting approaches. The presentations in session 4 reflected some of the large body of work that is ongoing.

Greg Keenan (Virent Energy Systems) presented an overview of the aqueous-phase reforming process for the conversion of sugars to hydrocarbons. This chemical catalytic process is an alternative to bio-based approaches for conversion. The extent to which the catalytic process can be applied to sugar mixtures derived from biomass will determine the versatility of this process for the generation of liquid fuels such as gasoline and other hydrocarbon-based liquid fuels. Selection of different catalysts and conditions allows conversion of sugars to different hydrocarbons and fuels. This process is currently being scaled up by Virent.

Andy Aden of National Renewable Energy Laboratory presented a paper titled "Comparison of Rigorous Process and Economic Data for Biochemical and Thermochemical Ethanol Production Technologies." A comparison of the biochemical approach (sugar platform) with the thermochemical approach for the conversion of lignocellulosic biomass into biofuels was made. Such a comparison will allow for a determination of the advantages/disadvantages of each approach. Capital and operating costs of each approach (thermochemical and biochemical) were compared. The results of this comparison suggested that the technology option of choice will be determined by the feedstock availability. There does not appear to be a clear technology winner when comparing these two options.

H. Blaschek ( $\square)$

University of Illinois at Urbana-Champaign, Urbana, IL, USA

e-mail: blaschek@uiuc.edu

A. Boateng

USDA-ARS, Eastern Regional Research Center, Wyndmoor, PA, USA 
Argonne National Laboratory's Seth Snyder presented "Membrane Contactor for Efficient Alcohol Recovery." Snyder provided an overview of the separative bioreactor technology for the recovery of organic acids and other charged products from fermentation broths. This work has been extended to include the recovery of alcohols and other neutral compounds. This latter approach employs ionic liquid extraction solvents. This approach has been used for recovery of ethanol and butanol. If scalable, this technology should significantly lower the recovery costs associated with the butanol fermentation.

Rathin Datta of Coskata Technologies presented a talk titled "Ethanol from Diverse Feedstocks-Coskata's Syngas Biotechnology Path." This discussion focused on the gasification of lignocellulosic biomass to syngas and the subsequent microbial conversion to ethanol. This technology depends on the use of novel microbes and engineering-based approaches for improving the mass transfer from the gaseous to the liquid phase. This technology is not new and has previously been presented at this meeting. However, this talk represented the commercial approach.

A talk on "Microbial Factories for the Production of Fit-For-Purpose Hydrocarbon Biofuels from Sugar Feedstocks" was presented by Neil Renninger of Amyris Biotechnologies. A synthetic biology-based approach involving microbial engineering for the production of hydrocarbon transportation fuels such as diesel, gasoline, and jet fuels was described.

Finally, William Provine of Dupont gave a talk on "Biobutanol-Next Generation Advanced Biofuel." The partnership between Dupont and BP with respect to biobutanol production was described. The advantages of biobutanol as a liquid fuel over ethanol were described in some detail. The difference between $n$-butanol and iso-butanol was also discussed with respect to the use of this chemical as a liquid fuel. An overview of butanol production in native and other microbes was briefly discussed. 